\title{
Perfluoroalkylated amphiphiles with a morpholinophosphate or a dimorpholinophosphate polar head group
}

\author{
Véronique M. Sadtler, ${ }^{a}$ François Jeanneaux, ${ }^{a}$ Marie Pierre Krafft, ${ }^{*, b}$ József Rábai $^{c}$ and \\ Jean G. Riess ${ }^{d}$ \\ a SOFT Intl., Unité de Chimie Moléculaire (CNRS URA 426), Parc Valrose, Université de \\ Nice-Sophia Antipolis, 06108 Nice cédex 2, France \\ ${ }^{b}$ Institut Charles Sadron (CNRS UPR 22), 6, rue Boussingault, 67083 Strasbourg cédex, France \\ ${ }^{c}$ Department of Organic Chemistry, Eötvös Loránd University, P.O. Box 32, Budapest 112, \\ H-1518 Hungary \\ ${ }^{d}$ University of California at San Diego Medical Center, 225 Dickinson St., San Diego, \\ CA 92103-1990, USA
}

\begin{abstract}
Some previously synthesized (perfluoroalkyl)alkyldimorpholinophosphates, $\mathrm{C}_{n} \mathrm{~F}_{2 n+1} \mathrm{C}_{m} \mathrm{H}_{2 m} \mathrm{OP}(\mathrm{O})$ $\left[\mathrm{N}\left(\mathrm{CH}_{2} \mathrm{CH}_{2}\right)_{2} \mathrm{O}\right]_{2}$, were found remarkably to stabilize heat sterilizable water-in-fluorocarbon reverse emulsions and to have a strong proclivity to self-aggregate into microtubular assemblies when dispersed in water. This series has now been extended in order to allow structure-property relationships to be established and product optimization to be achieved. A new series of even more fluorophilic compounds consisting in bis[(perfluoroalkyl)alkyl]monomorpholinophosphates, $\left(\mathrm{C}_{n} \mathrm{~F}_{2 n+1} \mathrm{C}_{m} \mathrm{H}_{2 m} \mathrm{O}\right)_{2} \mathrm{P}(\mathrm{O}) \mathrm{N}\left(\mathrm{CH}_{2} \mathrm{CH}_{2}\right)_{2} \mathrm{O}$, was also synthesized. Preliminary surfactant activity and biocompatibility data are presented and compared to data obtained with non-fluorinated analogues.
\end{abstract}

There is a need for novel, highly effective and biocompatible surfactants to serve as components of drug preparation and delivery systems. A large variety of well-defined pure perfluoroalkylated amphiphiles have been synthesized in recent years with this objective in mind ${ }^{1-4}$ and were investigated as emulsion stabilizers, vesicle-forming components and for other uses. ${ }^{5,6}$ Among the most fluorophilic neutral compounds synthesized were some (perfluoroalkyl)alkyldimorpholinophosphates, $\quad \mathrm{C}_{n} \mathrm{~F}_{2 n+1} \mathrm{C}_{m} \mathrm{H}_{2 m} \mathrm{OP}(\mathrm{O})\left[\mathrm{N}\left(\mathrm{CH}_{2} \mathrm{CH}_{2}\right)_{2} \mathrm{O}\right]_{2}$, (FnCmDMPs). ${ }^{7}$

Investigation of this initial series of compounds revealed several unexpectedly interesting features, including: (i) a remarkable capacity to allow the preparation and stabilization of water-in-fluorocarbon reverse emulsions; these emulsions, which were stable enough to withstand heat sterilization, could be loaded with a variety of drugs and other agents, and have potential in drug delivery through the pulmonary route ${ }^{8}$ (ii) a remarkable tendency to self-aggregate when dispersed in water and to yield sturdy, regularly shaped, long microtubules in which the surfactant molecules appear to be highly ordered; 9,10 when heated, these tubules reversibly convert into giant vesicles. When hydrocarbon analogues were dispersed in water no aggregates larger than micelles were seen, in line with the usual behaviour of single-chain amphiphiles; (iii) low acute toxicities, whether when administered intraperitoneally or intravascularly, as well as the absence of hemolytic activity in spite of their high surface activity. By contrast, the hydrogen analogues are highly hemolytic.

A more extensive series of dimorpholinophosphates was needed in order to optimize and establish these results on a firmer basis and to determine structure-property relationships. Compounds with higher and lower $\mathrm{Fn}: \mathrm{Cm}$ ratios

* Phone: +33 3884140 60. Fax: +33 3884140 99. E-mail: krafft@europe.u-strasbg.fr were synthesized as well as compounds with intermediate hydrocarbon spacer lengths, since such intermediate spacer lengths were shown to have an influence on the ordering of the surfactant films. ${ }^{11}$ In addition, the synthesis of even more fluorophilic compounds, with two perfluoroalkyl chains, was decided upon. These compounds consist in the bis(perfluoroalkyl)alkylmonomorpholinophosphates, $\left(\mathrm{C}_{n} \mathrm{~F}_{2 n+1} \mathrm{C}_{m} \mathrm{H}_{2 m} \mathrm{O}\right)_{2} \mathrm{P}(\mathrm{O}) \mathrm{N}\left(\mathrm{CH}_{2} \mathrm{CH}_{2}\right)_{2} \mathrm{O}$, code-named $\mathrm{D}(\mathrm{F} n \mathrm{Cm}) \mathrm{MMP}$. Some hydrogenated analogues were also prepared in order to determine the impact of the fluorinated chains on the amphiphiles' behaviour.

This paper describes the synthesis of the new $F$-alkylated mono- and dimorpholinophosphates, their characterization and a preliminary assessment of their surface activity and biocompatibility.

\section{Experimental}

\section{Materials and methods}

The perfluoroalkylated alcohols and iodides utilized were a gift from Atochem (Pierre-Bénite, France) or were synthesized as described below. They were recrystallised from chloroform or distilled under reduced pressure prior to use. The alcohols with a longer hydrocarbon spacer were synthesized according to Brace by radical addition of $\mathrm{C}_{n} \mathrm{~F}_{2 n+1} \mathrm{I}$ to terminally unsaturated alcohols $\mathrm{CH}_{2}=\mathrm{CH}\left(\mathrm{CH}_{2}\right)_{m-2} \mathrm{OH}$, followed by reduction with $\mathrm{HCl}-\mathrm{Zn}$ in anhydrous ethanol. ${ }^{12}$ (F-Heptyl)methanol, $\mathrm{C}_{7} \mathrm{~F}_{15} \mathrm{CH}_{2} \mathrm{OH}$, was prepared from the methyl perfluorooctanoate ester $\mathrm{C}_{7} \mathrm{~F}_{15} \mathrm{COOCH}_{3}$ by $\mathrm{NaBH}_{4}$ reduction in isopropanol. ${ }^{13}$ Synthesis of the ( $F$-alkyl)propanols is described elsewhere. ${ }^{14}$ Phosphorus oxytrichloride, triethylamine and morpholine (Aldrich) were distilled prior to use. Perfluorodecalin (FDC) was a gift from Air Products (USA).

TLC analyses were performed on RP-18 silica gel F-254 plates (Merck). Silica gel (70-230 mesh, Merck) was used for 
column chromatography. Melting points (uncorrected) were determined with a Reichert apparatus. IR spectra were recorded on a Bruker IFS 45 and NMR spectra on a Bruker AC 200 spectrometer (the FT-IR and NMR spectra of F6C3DMP, F7C1DMP, F8C3DMP and F10C3DMP were recorded on Bruker IFS 55 and Bruker $500 \mathrm{MHz}$ spectrometers by Dr. A. Csámpai, Hungary). The elemental analyses were performed by the Service Central de Microanalyses of the Centre National de la Recherche Scientifique (Lyon, France). Surface tensions, $\gamma_{s}$, and interfacial tensions, $\gamma_{i}$, were measured with an automatic Lauda tensiometer (platinum ring, Lecomte du Noüy method): aqueous solutions of the amphiphiles were prepared using deionized water (Elgastat UHQ system, $\gamma_{\mathrm{s}}=$ $72 \mathrm{mN} \mathrm{m}^{-1}$, and $\gamma_{\mathrm{i}}$ water/FDC $=56 \mathrm{mN} \mathrm{m}^{-1}$ ). Tension values were obtained after careful equilibration of the interface. Critical micellar concentrations $(\mathrm{cmc})$ were estimated from the well-marked breaking points of the $\gamma_{\mathrm{s}}-\log C$ curves.

\section{Biological tests}

In a typical cell culture test, ${ }^{15}$ ten flasks were filled with $2 \mathrm{~mL}$ of a suspension of $N_{1}$ Namalva lymphoblastoid cells in a nutrient medium; $2 \mathrm{~mL}$ of a $0.9 \% \mathrm{NaCl}$ solution of the perfluoroalkylated surfactant to be tested, or $2 \mathrm{~mL}$ of physiological water for the control series, was then added. After three days of incubation, the number of cells were counted $\left(N_{2}\right)$ and their viability determined by the Trypon Blue dye exclusion method: results were expressed as the ratio of living (noncolored) cells to the total number of cells.

The hemolytic activity was evaluated on human red blood cells according to ref. 16: $2 \mathrm{~mL}$ of a $1 \%$ suspension of human red blood cells were incubated for $1 \mathrm{~h}$ at $37^{\circ} \mathrm{C}$ with $2 \mathrm{~mL}$ of a $0.9 \% \mathrm{NaCl}$ solution of the surfactant to be tested. Detoxified water and physiological water were submitted to the same procedure and taken as a positive reference and a blank, respectively. After centrifugation, the optical density (OD) of the supernatant was measured at $540 \mathrm{~nm}$ and the percentage of hemolysis relative to detoxified water was calculated according to the relationship: $100\left(\mathrm{OD}_{\text {test }}-\mathrm{OD}_{\mathrm{NaCl}}\right) /\left(\mathrm{OD}_{\mathrm{H}_{2} \mathrm{O}}\right.$ $\left.-\mathrm{OD}_{\mathrm{NaCl}}\right)$.

The in vivo test consisted in injecting $0.5 \mathrm{~mL}$ of an isotonic solution of the surfactant to be tested into the tail vein (intravenous administration, i.v.) of 10 Dawley mice, weighing 20-25 g. Intraperitoneal administration (i.p) was achieved with a solution of surfactant in detoxified perfluorooctyl bromide. Growth, behaviour and any symptoms of intoxication of the animals were monitored over a period of one month and compared to those of a reference population of mice injected with the same volume of saline.

\section{Synthesis of the (perfluoroalkyl)alkyldimorpholinophosphates, FnCmDMPs}

[2-(F-Butyl)ethyl]dimorpholinophosphate, F4C2DMP. 2-(FButyl)ethanol (10.14 g, $38.4 \mathrm{mmol})$ and $14 \mathrm{~mL}$ of triethylamine ( 2.5 equiv) were dissolved in $50 \mathrm{~mL}$ of dry ether and added dropwise, at $0{ }^{\circ} \mathrm{C}$ and under anhydrous nitrogen, to $5.9 \mathrm{~g}(38.5$ $\mathrm{mmol}$ ) of phosphorus oxytrichloride in $70 \mathrm{~mL}$ of ether. The mixture was stirred at $0^{\circ} \mathrm{C}$ for $1 \mathrm{~h}$. [2-(F-Butyl)ethoxy]phosphoryl dichloride was identified as the major product $(>90 \%)$ of the reaction: ${ }^{1} \mathrm{H}$ NMR $\left(\delta, \mathrm{CDCl}_{3}\right): 2.57\left(\mathrm{tt}, 2 \mathrm{H},{ }^{3} J_{\mathrm{HH}}=5.5\right.$ $\left.\mathrm{Hz},{ }^{3} J_{\mathrm{HF}}=18 \mathrm{~Hz}, \mathrm{R}_{\mathrm{F}} \mathrm{CH}_{2}\right), 4.3\left(\mathrm{dt}, 2 \mathrm{H},{ }^{3} J_{\mathrm{HH}}=5.5 \mathrm{~Hz}\right.$, $\left.{ }^{3} J_{\mathrm{HP}}=7 \mathrm{~Hz}, \mathrm{CH}_{2} \mathrm{OP}\right) ;{ }^{31} \mathrm{P} \mathrm{NMR}\left(\delta, \mathrm{CDCl}_{3}\right):-6.1$, signal from by-product bis[2-(F-Butyl)ethoxy]phosphoryl monochloride: $\delta=-10$, less than $10 \%$ of the total signal area.

A solution of $6.68 \mathrm{~g}(76.7 \mathrm{mmol})$ of morpholine and $28 \mathrm{~mL}$ ( 2.5 equiv) of triethylamine in $50 \mathrm{~mL}$ of ether was then added dropwise to the stirred reaction mixture, which was maintained free from oxygen and cooled with an ice-bath. After stirring for $18 \mathrm{~h}$, the mixture was allowed to warm up to room temperature, triethylamine hydrochloride was filtered off, and the solvent and excess of amine were removed under vacuum. The clear oily residue was dried under reduced pressure and purified by chromatography (ethyl acetate-methanol $9: 1$ ), leading to $12.65 \mathrm{~g}$ of the final product $(69 \%$ with respect to the alcohol). Purity was controlled by TLC $\left(R_{\mathrm{f}}\right.$ value $=0.7$, ethyl acetate-methanol 9:1, 1\% fluorescein in ethanol solution spray); GC: $99.7 \%$. MW $=482 \mathrm{~g}$, liquid at $25^{\circ} \mathrm{C} .{ }^{1} \mathrm{H}$ NMR $(\delta$, TMS ref., $\mathrm{CDCl}_{3}$ ): 2.5 (tt, $2 \mathrm{H},{ }^{3} J_{\mathrm{HH}}=5.6 \mathrm{~Hz},{ }^{3} J_{\mathrm{HF}}=20.2 \mathrm{~Hz}$, $\left.\mathrm{R}_{\mathrm{F}} \mathrm{CH}_{2}\right), 3.1\left(\mathrm{td}, 8 \mathrm{H},{ }^{3} J_{\mathrm{HH}}=5.0 \mathrm{~Hz},{ }^{3} J_{\mathrm{HP}}=3.7 \mathrm{~Hz}, \mathrm{NCH}_{2}\right), 3.6$ $\left(\mathrm{t}, 8 \mathrm{H},{ }^{3} J_{\mathrm{HH}}=5.1 \mathrm{~Hz}, \mathrm{CH}_{2} \mathrm{OCH}_{2}\right), 4.2\left(\mathrm{td}, 2 \mathrm{H},{ }^{3} J_{\mathrm{HH}}=6.0 \mathrm{~Hz}\right.$, $\left.{ }^{3} J_{\mathrm{HP}}=7.6 \mathrm{~Hz}, \mathrm{CH}_{2} \mathrm{OP}\right) ;{ }^{19} \mathrm{~F} \mathrm{NMR}\left(\delta, \mathrm{CFCl}_{3}\right.$ ref., $\left.\mathrm{CDCl}_{3}\right)$ : $-81.5\left(\mathrm{CF}_{3}\right),-114.3\left(\mathrm{CF}_{2} \mathrm{CH}_{2}\right),-125.0\left(\mathrm{CF}_{3} \mathrm{CF}_{2} \mathrm{CF}_{2}\right)$, $-126.5\left(\mathrm{CF}_{3} \mathrm{CF}_{2}\right) ;{ }^{13} \mathrm{C}$ NMR ( $\delta$, TMS ref., $\left.\mathrm{CDCl}_{3}\right): 32.1$ (td, $\left.{ }^{2} J_{\mathrm{CF}}=21.5 \mathrm{~Hz},{ }^{3} J_{\mathrm{CP}}=7.1 \mathrm{~Hz}, \mathrm{R}_{\mathrm{F}} \mathrm{CH}_{2}\right), 44.6\left(\mathrm{~s}, \mathrm{NCH}_{2}\right), 57.2$ $\left(\mathrm{d},{ }^{2} J_{\mathrm{PC}}=4.2 \mathrm{~Hz}, \quad \mathrm{CH}_{2} \mathrm{OP}\right), \quad 67.1 \quad\left(\mathrm{~d}, \quad{ }^{3} J_{\mathrm{CP}}=6.5 \quad \mathrm{~Hz}\right.$, $\left.\mathrm{CH}_{2} \mathrm{OCH}_{2}\right) ;{ }^{31} \mathrm{P}$ NMR $\left(\delta, \mathrm{H}_{3} \mathrm{PO}_{4}\right.$ ref., $\left.\mathrm{CDCl}_{3}\right): 14.1 ; \mathrm{IR}(\mathrm{v} /$ $\left.\mathrm{cm}^{-1}\right)$ : 1250-1180 ( $\left.\mathrm{P}=\mathrm{O}, \mathrm{CF}\right), 971(\mathrm{PN})$. Anal. calcd $(\%)$ for $\mathrm{C}_{14} \mathrm{H}_{20} \mathrm{~F}_{9} \mathrm{~N}_{2} \mathrm{O}_{4} \mathrm{P}$ : C 34.85; $\mathrm{H} \mathrm{4.15;} \mathrm{N} \mathrm{5.81,} \mathrm{P} \mathrm{6.43;} \mathrm{found} \mathrm{C}$ $34.64 ; \mathrm{H} 4.13$; N 5.73; P 6.29 .

The same procedure was applied for the synthesis of all dimorpholinophosphates using the quantities mentioned below.

[3-(F-Hexyl)propyl] dimorpholinophosphate, F6C3DMP. 3-(F-Hexyl)propanol (21.2 mmol), $21.2 \mathrm{mmol}$ of $\mathrm{OPCl}_{3}$ and 53 mmol of morpholine. Yield $=69 \%$ after chromatography (ether-chloroform-methanol $4.5: 5: 0.5$ ) and recrystallisation in isooctane. $\mathrm{MW}=596 \mathrm{~g}, \mathrm{~m} . \mathrm{p} .=40^{\circ} \mathrm{C} .{ }^{1} \mathrm{H}$ NMR $(\delta$, $\left.\mathrm{CDCl}_{3}\right): 1.9\left(\mathrm{~m}, 2 \mathrm{H}, \mathrm{R}_{\mathrm{F}} \mathrm{CH}_{2}\right), 2.1\left(\mathrm{~m}, 2 \mathrm{H}, \mathrm{R}_{\mathrm{F}} \mathrm{CH}_{2} \mathrm{CH}_{2}\right), 3.1$ (td, $\left.8 \mathrm{H},{ }^{3} J_{\mathrm{HH}}=6.6 \mathrm{~Hz},{ }^{3} J_{\mathrm{HP}}=6.7 \mathrm{~Hz}, \mathrm{NCH}_{2}\right), 3.6\left(\mathrm{t}, 8 \mathrm{H},{ }^{3} J_{\mathrm{HH}}=\right.$ $\left.6.3 \mathrm{~Hz}, \mathrm{CH}_{2} \mathrm{OCH}_{2}\right), 4.0\left(\mathrm{td}, 2 \mathrm{H},{ }^{3} J_{\mathrm{HH}}=7.8 \mathrm{~Hz},{ }^{3} J_{\mathrm{HP}}=9.8 \mathrm{~Hz}\right.$, $\left.\mathrm{CH}_{2} \mathrm{OP}\right) .{ }^{13} \mathrm{C}$ NMR $\left(\delta, \mathrm{CDCl}_{3}\right): 21.9\left(\mathrm{~s}, \mathrm{R}_{\mathrm{F}} \mathrm{CH}_{2}\right), 27.7(\mathrm{t}$, $\left.\mathrm{R}_{\mathrm{F}} \mathrm{CH}_{2} \mathrm{CH}_{2}\right) 44.8\left(\mathrm{~s}, \mathrm{NCH}_{2}\right), 63.9\left(\mathrm{~d},{ }^{2} J_{\mathrm{CP}}=5.0 \mathrm{~Hz}, \mathrm{CH}_{2} \mathrm{OP}\right)$, $67.2\left(\mathrm{~d},{ }^{3} J_{\mathrm{CP}}=5.5 \mathrm{~Hz}, \mathrm{CH}_{2} \mathrm{OCH}_{2}\right) .{ }^{31} \mathrm{P} \mathrm{NMR}\left(\delta, \mathrm{CDCl}_{3}\right)$ : 14.8.

[1-(F-Heptyl)methyl] dimorpholinophosphate, F7C1DMP. 1-(F-Heptyl)methanol $(10.0 \mathrm{mmol}), 10.0 \mathrm{mmol}$ of $\mathrm{POCl}_{3}$ and $25 \mathrm{mmol}$ of morpholine. Yield $=60 \%$. MW $=618 \mathrm{~g}$, m.p. $=49{ }^{\circ} \mathrm{C} .{ }^{1} \mathrm{H} \mathrm{NMR}\left(\delta, \mathrm{CDCl}_{3}\right): 3.1\left(\mathrm{td}, 8 \mathrm{H},{ }^{3} J_{\mathrm{HH}}=5.0 \mathrm{~Hz}\right.$, $\left.{ }^{3} J_{\mathrm{HP}}=5.8 \mathrm{~Hz}, \mathrm{NCH}_{2}\right), 3.7\left(\mathrm{t}, 8 \mathrm{H},{ }^{3} J_{\mathrm{HH}}=4.5 \mathrm{~Hz}, \mathrm{CH}_{2} \mathrm{OCH}_{2}\right)$, $4.4\left(\mathrm{td}, 2 \mathrm{H},{ }^{3} J_{\mathrm{HH}}=6.7 \mathrm{~Hz},{ }^{3} J_{\mathrm{HP}}=13.3 \mathrm{~Hz}, \mathrm{CH}_{2} \mathrm{OP}\right) .{ }^{13} \mathrm{C}$ $\operatorname{NMR}\left(\delta, \mathrm{CDCl}_{3}\right): 44.7\left(\mathrm{~s}, \mathrm{NCH}_{2}\right), 61.7\left(\mathrm{t}, \mathrm{CH}_{2} \mathrm{OP}\right), 67.1(\mathrm{~s}$, $\left.\mathrm{CH}_{2} \mathrm{OCH}_{2}\right) .{ }^{31} \mathrm{P}$ NMR $\left(\delta, \mathrm{CDCl}_{3}\right): 15.5$. IR $\left(\mathrm{v} / \mathrm{cm}^{-1}\right): 1240-$ $1210(\mathrm{CF}), 2890-2850(\mathrm{CH}), 974(\mathrm{PN})$.

[3-(F-Octyl)propyl] dimorpholinophosphate, F8C3DMP. 3-(F-Octyl)propanol $(12.5 \mathrm{mmol}), 12.5 \mathrm{mmol}$ of $\mathrm{OPCl}_{3}$ and $31.4 \mathrm{mmol}$ of morpholine. Yield $=64 \%$ after chromatography (ether-chloroform-methanol $4.5: 5: 0.5$ ) and recrystallisation in isooctane. $\mathrm{MW}=696 \mathrm{~g}, \mathrm{~m} . \mathrm{p} .=59{ }^{\circ} \mathrm{C} .{ }^{1} \mathrm{H}$ NMR $(\delta$, $\left.\mathrm{CDCl}_{3}\right): 2.0\left(\mathrm{~m}, 2 \mathrm{H}, \mathrm{R}_{\mathrm{F}} \mathrm{CH}_{2}\right), 2.2\left(\mathrm{~m}, 2 \mathrm{H}, \mathrm{R}_{\mathrm{F}} \mathrm{CH}_{2} \mathrm{CH}_{2}\right), 3.1(\mathrm{td}$, $\left.8 \mathrm{H},{ }^{3} J_{\mathrm{HH}}=6.6 \mathrm{~Hz},{ }^{3} J_{\mathrm{HP}}=6.7 \mathrm{~Hz}, \mathrm{NCH}_{2}\right), 3.6\left(\mathrm{t}, 8 \mathrm{H},{ }^{3} J_{\mathrm{HH}}=\right.$ $\left.6.3 \mathrm{~Hz}, \mathrm{CH}_{2} \mathrm{OCH}_{2}\right), 4.0\left(\mathrm{td}, 2 \mathrm{H},{ }^{3} J_{\mathrm{HH}}=7.8 \mathrm{~Hz},{ }^{3} J_{\mathrm{HP}}=9.8 \mathrm{~Hz}\right.$, $\left.\mathrm{CH}_{2} \mathrm{OP}\right) .{ }^{13} \mathrm{C} \mathrm{NMR}\left(\delta, \mathrm{CDCl}_{3}\right): 21.9\left(\mathrm{~s}, \mathrm{R}_{\mathrm{F}} \mathrm{CH}_{2}\right), 27.7(\mathrm{t}$, $\left.\mathrm{R}_{\mathrm{F}} \mathrm{CH}_{2} \mathrm{CH}_{2}\right), 44.8\left(\mathrm{~s}, \mathrm{NCH}_{2}\right), 63.9\left(\mathrm{~d},{ }^{2} J_{\mathrm{CP}}=5.0 \mathrm{~Hz}, \mathrm{CH}_{2} \mathrm{OP}\right)$, $67.2\left(\mathrm{~d},{ }^{3} J_{\mathrm{CP}}=5.5 \mathrm{~Hz}, \mathrm{CH}_{2} \mathrm{OCH}_{2}\right) .{ }^{31} \mathrm{P} \mathrm{NMR}\left(\delta, \mathrm{CDCl}_{3}\right)$ : 14.8.

[5-(F-Octyl)pentyl] dimorpholinophosphate,

F8C5DMP. 5-(F-Octyl)pentanol (32.6 mmol), $32.6 \mathrm{mmol}$ of $\mathrm{OPCl}_{3}$ and $70.4 \mathrm{mmol}$ of morpholine. Yield $=73 \%$. MW $=724 \mathrm{~g}$; m.p. $=78^{\circ} \mathrm{C} .{ }^{1} \mathrm{H}$ NMR $\left(\delta, \mathrm{CDCl}_{3}\right): 1.5(\mathrm{~s}, 2 \mathrm{H}), 1.7(\mathrm{~m}, 4 \mathrm{H}), 2.1$ $\left(\mathrm{tt}, 2 \mathrm{H},{ }^{3} J_{\mathrm{HH}}=6.2 \mathrm{~Hz},{ }^{3} J_{\mathrm{HF}}=19.1 \mathrm{~Hz}, \mathrm{R}_{\mathrm{F}} \mathrm{CH}_{2}\right), 3.1(\mathrm{td}, 8 \mathrm{H}$, 
$\left.{ }^{3} J_{\mathrm{HH}}=6.1 \mathrm{~Hz},{ }^{3} J_{\mathrm{HP}}=4.5 \mathrm{~Hz}, \mathrm{NCH}_{2}\right), 3.6\left(\mathrm{t}, 8 \mathrm{H},{ }^{3} J_{\mathrm{HH}}=6.1\right.$ $\left.\mathrm{Hz}, \mathrm{CH}_{2} \mathrm{OCH}_{2}\right), 3.9\left(\mathrm{td}, 2 \mathrm{H},{ }^{3} J_{\mathrm{HH}}=6.2 \mathrm{~Hz},{ }^{3} J_{\mathrm{HP}}=6.9 \mathrm{~Hz}\right.$, $\left.\mathrm{CH}_{2} \mathrm{OP}\right) .{ }^{19} \mathrm{~F}$ NMR $\left(\delta, \mathrm{CDCl}_{3}\right):-81.2\left(\mathrm{CF}_{3}\right),-114.8$ $\left(\mathrm{CF}_{2} \mathrm{CH}_{2}\right),-122.3(6 \mathrm{~F}),-123.2(2 \mathrm{~F}),-123.9(2 \mathrm{~F}),-126.6$ $\left(\mathrm{CF}_{3} \mathrm{CF}_{2}\right) \cdot{ }^{13} \mathrm{C}$ NMR $\left(\delta, \mathrm{CDCl}_{3}\right): 20.1\left(\mathrm{t},{ }^{3} J_{\mathrm{CF}}=5.5 \mathrm{~Hz}\right.$, $\left.\mathrm{R}_{\mathrm{F}} \mathrm{CH}_{2} \mathrm{CH}_{2}\right), 25.5,30.3\left(\mathrm{~s}, 2 \mathrm{CH}_{2}\right), 31.1\left(\mathrm{t},{ }^{2} J_{\mathrm{CF}}=21.5 \mathrm{~Hz}\right.$, $\left.\mathrm{R}_{\mathrm{F}} \mathrm{CH}_{2}\right), 44.9\left(\mathrm{~s}, \mathrm{NCH}_{2}\right), 65.2\left(\mathrm{~d},{ }^{2} J_{\mathrm{CP}}=3.8 \mathrm{~Hz}, \mathrm{CH}_{2} \mathrm{OP}\right), 67.3$ $\left(\mathrm{d},{ }^{3} J_{\mathrm{CP}}=5.5 \mathrm{~Hz}, \mathrm{CH}_{2} \mathrm{OCH}_{2}\right) .{ }^{31} \mathrm{P} \mathrm{NMR}\left(\delta, \mathrm{CDCl}_{3}\right): 13.7$. IR $\left(\mathrm{v} / \mathrm{cm}^{-1}\right): 1250-1150(\mathrm{P}=\mathrm{O}, \mathrm{CF}), 974(\mathrm{PN}), 2930-2850(\mathrm{CH})$. Anal. calcd (\%) for $\mathrm{C}_{21} \mathrm{H}_{26} \mathrm{~F}_{17} \mathrm{~N}_{2} \mathrm{O}_{4} \mathrm{P}$ : C 34.81; $\mathrm{H} 3.59 ; \mathrm{N}$ 3.87; P 4.28; found: C 34.64; H 3.74; N 3.89; P 4.35.

[1-(F-Nonyl)methyl] dimorpholinophosphate, F9C1DMP. 1-(F-Nonyl)methanol $(8.8 \mathrm{mmol}), 8.9 \mathrm{mmol}$ of $\mathrm{OPCl}_{3}$ and 17.7 mmol of morpholine. Yield $=71 \% . \quad M W=718 \quad \mathrm{~g}$, m.p. $=67{ }^{\circ} \mathrm{C} .{ }^{1} \mathrm{H} \mathrm{NMR}\left(\delta, \mathrm{CDCl}_{3}\right): 3.1\left(\mathrm{td}, 8 \mathrm{H},{ }^{3} J_{\mathrm{HH}}=6.1 \mathrm{~Hz}\right.$, $\left.{ }^{3} J_{\mathrm{HP}}=3.1 \mathrm{~Hz}, \mathrm{NCH}_{2}\right), 3.6\left(\mathrm{t}, 8 \mathrm{H},{ }^{3} J_{\mathrm{HH}}=6 \mathrm{~Hz}, \mathrm{CH}_{2} \mathrm{OCH}_{2}\right)$, $4.4\left(\mathrm{td}, 2 \mathrm{H},{ }^{3} J_{\mathrm{HF}}=22.7 \mathrm{~Hz},{ }^{3} J_{\mathrm{HP}}=7.5 \mathrm{~Hz}, \mathrm{CH}_{2} \mathrm{OP}\right) .{ }^{19} \mathrm{~F}$ $\operatorname{NMR}\left(\delta, \mathrm{CDCl}_{3}\right):-81.2\left(\mathrm{CF}_{3}\right),-120.7\left(\mathrm{CF}_{2} \mathrm{CH}_{2}\right),-122.3$ (4F), -123.1 (2F), - $123.5(6 \mathrm{~F}),-126.6\left(\mathrm{CF}_{3} \mathrm{CF}_{2}\right) \cdot{ }^{13} \mathrm{C} \mathrm{NMR}$ $\left(\delta, \mathrm{CDCl}_{3}\right): 44.8\left(\mathrm{~s}, \mathrm{NCH}_{2}\right), 61.8\left(\mathrm{dt},{ }^{2} J_{\mathrm{CF}}=20.0 \mathrm{~Hz},{ }^{2} J_{\mathrm{CP}}=\right.$ $\left.4.0 \mathrm{~Hz}, \mathrm{CH}_{2} \mathrm{OP}\right), 67.1\left(\mathrm{~d},{ }^{3} J_{\mathrm{CP}}=5.5 \mathrm{~Hz}, \mathrm{CH}_{2} \mathrm{OCH}_{2}\right) .{ }^{31} \mathrm{P}$ NMR $\left(\delta, \mathrm{CDCl}_{3}\right): 14.7$ IR $\left(\mathrm{v} / \mathrm{cm}^{-1}\right): 1250-1150(\mathrm{P}=\mathrm{O}, \mathrm{CF})$, 976 (PN). Anal. calcd (\%) for $\mathrm{C}_{18} \mathrm{H}_{18} \mathrm{~F}_{19} \mathrm{~N}_{2} \mathrm{O}_{4} \mathrm{P}: \mathrm{C} 30.08 ; \mathrm{H}$ 2.52 ; N 3.9; P 4.32; found: C 30.21, H 2.34; N 3.85; P 4.38.

[2-(F-Decyl)ethyl] dimorpholinophosphate, F10C2DMP. 2-(F-Decyl)ethanol (18.4 mmol), $18.4 \mathrm{mmol}$ of $\mathrm{OPCl}_{3}$ and 36.9 mmol of morpholine. Yield $=65 \%$. GC: $99.7 \%$. MW $=782 \mathrm{~g}$, m.p. $=91{ }^{\circ} \mathrm{C} .{ }^{1} \mathrm{H}$ NMR $\left(\delta, \mathrm{CDCl}_{3}\right): 2.5\left(\mathrm{tt}, 2 \mathrm{H},{ }^{3} J_{\mathrm{HH}}=7.8 \mathrm{~Hz}\right.$, $\left.{ }^{3} J_{\mathrm{HF}}=19.6 \mathrm{~Hz}, \mathrm{R}_{\mathrm{F}} \mathrm{CH}_{2}\right), 3.1\left(\mathrm{td}, 8 \mathrm{H},{ }^{3} J_{\mathrm{HH}}=6.6 \mathrm{~Hz},{ }^{3} J_{\mathrm{HP}}=\right.$ $\left.6.7 \mathrm{~Hz}, \mathrm{NCH}_{2}\right), 3.6\left(\mathrm{t}, 8 \mathrm{H},{ }^{3} J_{\mathrm{HH}}=6.3 \mathrm{~Hz}, \mathrm{CH}_{2} \mathrm{OCH}_{2}\right), 4.3(\mathrm{td}$, $\left.2 \mathrm{H},{ }^{3} J_{\mathrm{HH}}=7.8 \mathrm{~Hz},{ }^{3} J_{\mathrm{HP}}=9.8 \mathrm{~Hz}, \mathrm{CH}_{2} \mathrm{OP}\right) .{ }^{19} \mathrm{~F} \operatorname{NMR}(\delta$, $\left.\mathrm{CDCl}_{3}\right):-81.3\left(\mathrm{CF}_{3}\right),-114.1\left(\mathrm{CF}_{2} \mathrm{CH}_{2}\right),-122.3(10 \mathrm{~F})$, $-123.2(2 \mathrm{~F}),-124.1(2 \mathrm{~F}),-126.7\left(\mathrm{CF}_{3} \mathrm{CF}_{2}\right) .{ }^{13} \mathrm{C}$ NMR $(\delta$, $\left.\mathrm{CDCl}_{3}\right): 32.3\left(\mathrm{td},{ }^{2} J_{\mathrm{CF}}=21.3 \mathrm{~Hz},{ }^{3} J_{\mathrm{CP}}=5.7 \mathrm{~Hz}, \mathrm{R}_{\mathrm{F}} \mathrm{CH}_{2}\right), 44.8$ $\left(\mathrm{s}, \mathrm{NCH}_{2}\right), 57.4\left(\mathrm{~d},{ }^{2} J_{\mathrm{CP}}=5.0 \mathrm{~Hz}, \mathrm{CH}_{2} \mathrm{OP}\right), 67.1\left(\mathrm{~d},{ }^{3} J_{\mathrm{CP}}=5.5\right.$ $\left.\mathrm{Hz}, \mathrm{CH}_{2} \mathrm{OCH}_{2}\right) .{ }^{31} \mathrm{P} \mathrm{NMR}\left(\delta, \mathrm{CDCl}_{3}\right): 14.2$. IR $\left(\mathrm{v} / \mathrm{cm}^{-1}\right)$ : 1250-1150 (P=O, CF), $978(\mathrm{PN})$. Anal. calcd $(\%)$ for $\mathrm{C}_{20} \mathrm{H}_{20} \mathrm{~F}_{21} \mathrm{~N}_{2} \mathrm{O}_{4} \mathrm{P}$ : C 30.69; H 2.56; N 1.79; P 3.96; found: $\mathrm{C}$ $30.74 ; \mathrm{H} 2.46$; N 1.82 ; P 3.85 .

[3-(F-Decyl)propyl] dimorpholinophosphate, F10C3DMP. 3-(F-Decyl)propanol (10.4 mmol), $10.4 \mathrm{mmol}$ of $\mathrm{OPCl}_{3}$ and 26 mmol of morpholine. Yield $=52 \%$ after chromatography (ether-chloroform-methanol $4.5: 5: 0.5$ ) and recrystallisation in isooctane. $\mathrm{MW}=796 \mathrm{~g}, \mathrm{~m} . \mathrm{p} .=89^{\circ} \mathrm{C} .{ }^{1} \mathrm{H} \operatorname{NMR}(\delta$, $\left.\mathrm{CDCl}_{3}\right): 1.9\left(\mathrm{~m}, 2 \mathrm{H}, \mathrm{R}_{\mathrm{F}} \mathrm{CH}_{2}\right), 2.2\left(\mathrm{~m}, 2 \mathrm{H}, \mathrm{R}_{\mathrm{F}} \mathrm{CH}_{2} \mathrm{CH}_{2}\right), 3.1$ (td, $\left.8 \mathrm{H},{ }^{3} J_{\mathrm{HH}}=6.6 \mathrm{~Hz},{ }^{3} J_{\mathrm{HP}}=6.7 \mathrm{~Hz}, \mathrm{NCH}_{2}\right), 3.6\left(\mathrm{t}, 8 \mathrm{H},{ }^{3} J_{\mathrm{HH}}=\right.$ $\left.6.3 \mathrm{~Hz}, \mathrm{CH}_{2} \mathrm{OCH}_{2}\right), 4.1\left(\mathrm{td}, 2 \mathrm{H},{ }^{3} J_{\mathrm{HH}}=7.8 \mathrm{~Hz},{ }^{3} J_{\mathrm{HP}}=9.8 \mathrm{~Hz}\right.$, $\left.\mathrm{CH}_{2} \mathrm{OP}\right) .{ }^{13} \mathrm{C} \mathrm{NMR}\left(\delta, \mathrm{CDCl}_{3}\right): 21.9\left(\mathrm{~s}, \mathrm{R}_{\mathrm{F}} \mathrm{CH}_{2}\right), 27.7(\mathrm{t}$, $\left.\mathrm{R}_{\mathrm{F}} \mathrm{CH}_{2} \mathrm{CH}_{2}\right), 44.8\left(\mathrm{~s}, \mathrm{NCH}_{2}\right), 63.9\left(\mathrm{~d},{ }^{2} J_{\mathrm{CP}}=5.0 \mathrm{~Hz}, \mathrm{CH}_{2} \mathrm{OP}\right)$, $67.2\left(\mathrm{~d},{ }^{3} J_{\mathrm{CP}}=5.5 \mathrm{~Hz}, \mathrm{CH}_{2} \mathrm{OCH}_{2}\right) .{ }^{31} \mathrm{P} \mathrm{NMR}\left(\delta, \mathrm{CDCl}_{3}\right)$ : 14.8.

Pentadecyldimorpholinophosphate, C15DMP. Pentadecanol (25.5 mmol), $25.6 \mathrm{mmol}$ of $\mathrm{OPCl}_{3}$ and $51.1 \mathrm{mmol}$ of morpholine. Yield $=65 \%$. MW $=446 \mathrm{~g}, \mathrm{~m} . \mathrm{p} .=45^{\circ} \mathrm{C} .{ }^{1} \mathrm{H}$ NMR $(\delta$, $\left.\mathrm{CDCl}_{3}\right): 0.8\left(\mathrm{t}, \mathrm{CH}_{3}\right), 1.2(\mathrm{~m}, 24 \mathrm{H}), 1.6\left(\mathrm{~m}, \mathrm{CH}_{2} \mathrm{CH}_{2} \mathrm{OP}\right), 3.1$ $\left(\mathrm{td}, 8 \mathrm{H},{ }^{3} J_{\mathrm{HH}}=6.2 \mathrm{~Hz},{ }^{3} J_{\mathrm{HP}}=2.7 \mathrm{~Hz}, \mathrm{NCH}_{2}\right), 3.6(\mathrm{t}, 8 \mathrm{H}$, $\left.{ }^{3} J_{\mathrm{HH}}=6.2 \mathrm{~Hz}, \mathrm{CH}_{2} \mathrm{OCH}_{2}\right), 3.9\left(\mathrm{dt}, 2 \mathrm{H},{ }^{3} J_{\mathrm{HH}}=6.6 \mathrm{~Hz}\right.$, $\left.{ }^{3} J_{\mathrm{HP}}=6.9 \mathrm{~Hz}, \mathrm{CH}_{2} \mathrm{OP}\right) .{ }^{13} \mathrm{C} \mathrm{NMR}\left(\delta, \mathrm{CDCl}_{3}\right): 14.2\left(\mathrm{~s}, \mathrm{CH}_{3}\right)$,
22.8, 25.8, 29.3, 29.4, 29.6, 29.7, 32.0 (all s, $12 \mathrm{CH}_{2}$ ), 30.6 (d, $\left.{ }^{3} J_{\mathrm{CP}}=6 \mathrm{~Hz}, \mathrm{CH}_{2} \mathrm{CH}_{2} \mathrm{O}\right), 44.8\left(\mathrm{~s}, \mathrm{NCH}_{2}\right), 65.7\left(\mathrm{~d},{ }^{2} J_{\mathrm{CP}}=5.5\right.$ $\left.\mathrm{Hz}, \mathrm{CH}_{2} \mathrm{OP}\right), 67.3\left(\mathrm{~d},{ }^{3} J_{\mathrm{CP}}=5.7 \mathrm{~Hz}, \mathrm{CH}_{2} \mathrm{OCH}_{2}\right) .{ }^{31} \mathrm{P} \mathrm{NMR}$ $\left(\delta, \mathrm{CDCl}_{3}\right)$ : 13.7. IR $\left(\mathrm{v} / \mathrm{cm}^{-1}\right): 1260-1230(\mathrm{P}=\mathrm{O}), 962(\mathrm{PN})$, 2930-2850 (CH). Anal. calcd (\%) for $\mathrm{C}_{23} \mathrm{H}_{47} \mathrm{~N}_{2} \mathrm{O}_{4} \mathrm{P}$ : C 31.88; $\mathrm{H} 10.54$; N 6.28; P 6.95; found: C 31.95; H 10.68; N 6.35; P 7.02 .

\section{Synthesis of the bis(perfluoroalkyl) \\ monomorpholinophosphates, D(FnCm)MMPs}

Bis [2-(F-hexyl)ethyl]monomorpholinophosphate, D(F6C2)MMP. $\mathrm{OPCl}_{3}(8.38 \mathrm{~g}, 54.6 \mathrm{mmol})$ was dissolved in $100 \mathrm{ml}$ of dry ether under dry nitrogen. 2-(F-Hexyl)ethanol (39.9 g, 109.6 $\mathrm{mmol})$ and $10.2 \mathrm{~g}(14 \mathrm{~mL}, 112 \mathrm{mmol}) \mathrm{of}^{\mathrm{NEt}_{3}}$ in $50 \mathrm{~mL}$ of $\mathrm{Et}_{2} \mathrm{O}$ were added dropwise to this solution at $50^{\circ} \mathrm{C}$. The mixture was stirred for $12 \mathrm{~h}$ at room temperature, filtered and the solvent and the excess of amine were removed under vacuum. Morpholine (10.1 g, $116 \mathrm{mmol})$ in $50 \mathrm{~mL} \mathrm{Et}_{2} \mathrm{O}$ was then added dropwise at $0{ }^{\circ} \mathrm{C}$. The reaction mixture was refluxed and stirred for $12 \mathrm{~h}$. The precipitate was then filtered, washed with $\mathrm{Et}_{2} \mathrm{O}$ and the solvent evaporated. The crude product was purified by column chromatography on silica (gradient from $10 \%$ ethyl acetate in hexane to $100 \%$ ethyl acetate). Recrystallisation from hexane yielded $30.4 \mathrm{~g}(65 \%$ with respect to the fluorinated alcohol) of the final product. $\mathrm{MW}=859 \mathrm{~g}, \mathrm{~m} . \mathrm{p} .=48{ }^{\circ} \mathrm{C} .{ }^{1} \mathrm{H}$ NMR $\left(\delta, \mathrm{CDCl}_{3}\right): 2.45(\mathrm{~m}, 4 \mathrm{H}$, $\left.\mathrm{R}_{\mathrm{F}} \mathrm{CH}_{2}\right), 3.09\left(\mathrm{~m}, 4 \mathrm{H}, \mathrm{CH}_{2} \mathrm{~N}\right), 3.6\left(\mathrm{~m}, 4 \mathrm{H}, \mathrm{CH}_{2} \mathrm{OCH}_{2}\right), 4.25$ $\left[\mathrm{m}, 4 \mathrm{H},\left(\mathrm{CH}_{2} \mathrm{O}\right)_{2} \mathrm{PO}\right] .{ }^{19} \mathrm{~F}$ NMR $\left(\delta, \mathrm{CDCl}_{3}\right):-81.3\left(\mathrm{CF}_{3}\right)$, $-114.0\left(\mathrm{CF}_{2} \mathrm{CH}_{2}\right),-122.4(2 \mathrm{~F}),-123.4(2 \mathrm{~F}),-124.1(2 \mathrm{~F})$, $-126.6\left(\mathrm{CF}_{3} \mathrm{CF}_{2}\right) .{ }^{31} \mathrm{P}$ NMR $\left(\delta, \mathrm{CDCl}_{3}\right): 8.34$. IR $\left(\mathrm{v} / \mathrm{cm}^{-1}\right)$ : 1250-1150 $(\mathrm{P}=\mathrm{O}, \mathrm{CF}), 970(\mathrm{PN})$. Anal. calcd $(\%)$ for $\mathrm{C}_{20} \mathrm{H}_{16} \mathrm{~F}_{26} \mathrm{NO}_{4} \mathrm{P}$ : C 27.95; H 1.88; N 1.63; P 1.64; found: $\mathrm{C}$ $27.91 ; \mathrm{H} 1.82 ; \mathrm{N} 1.59 ; \mathrm{P} 1.60$.

Bis [2-(F-octyl)ethyl] monomorpholinophosphate, D(F8C2)MMP. The same procedure was applied to $6.91 \mathrm{~g}(45.0 \mathrm{mmol})$ of $\mathrm{OPCl}_{3}, 41.82 \mathrm{~g}(90.2 \mathrm{mmol})$ of 2 -(F-octyl)ethanol and 11.3 $\mathrm{mL}(8.51 \mathrm{~g}, 90.3 \mathrm{mmol})$ of $\mathrm{NEt}_{3}$. The reaction mixture, after having been stirred for $12 \mathrm{~h}$ at room temperature, was refluxed for $4 \mathrm{~h}$. Morpholine $(7.93 \mathrm{~g}, 91.1 \mathrm{mmol})$ was then added at room temperature and the mixture was refluxed for $12 \mathrm{~h}$ and filtered. The precipitate was extracted with a $\mathrm{CHCl}_{3}-\mathrm{H}_{2} \mathrm{O}$ mixture. The ether and chloroform solutions were removed and the combined residues were chromatographed on silica with the same gradient as above. Recrystallisation from hexane yielded $24.3 \mathrm{~g}(55 \%)$ of a white material. $\mathrm{MW}=1059 \mathrm{~g}, \mathrm{~m} . \mathrm{p} .=79^{\circ} \mathrm{C},{ }^{1} \mathrm{H} \operatorname{NMR}\left(\delta, \mathrm{CDCl}_{3}\right)$ : $2.55\left(\mathrm{~m}, 4 \mathrm{H}, \mathrm{R}_{\mathrm{F}} \mathrm{CH}_{2}\right), 3.19\left(\mathrm{~m}, 4 \mathrm{H}, \mathrm{CH}_{2} \mathrm{~N}\right), 3.7(\mathrm{~m}, 4 \mathrm{H}$, $\left.\mathrm{CH}_{2} \mathrm{OCH}_{2}\right), 4.33\left[\mathrm{~m}, 4 \mathrm{H},\left(\mathrm{CH}_{2} \mathrm{O}\right)_{2} \mathrm{PO}\right] .{ }^{19} \mathrm{~F} \operatorname{NMR}(\delta$, $\left.\mathrm{CDCl}_{3}\right):-81.3\left(\mathrm{CF}_{3}\right),-114.2\left(\mathrm{CF}_{2} \mathrm{CH}_{2}\right),-122.5(4 \mathrm{~F})$, $-123.4(4 \mathrm{~F}),-124.1(2 \mathrm{~F}),-126.6\left(\mathrm{CF}_{3} \mathrm{CF}_{2}\right) .{ }^{31} \mathrm{P}$ NMR $(\delta$, $\left.\mathrm{CDCl}_{3}\right): 8.29$. IR $\left(\mathrm{v} / \mathrm{cm}^{-1}\right): 1250-1150(\mathrm{P}=\mathrm{O}, \mathrm{CF}), 970(\mathrm{PN})$. Anal. calcd (\%) for $\mathrm{C}_{24} \mathrm{H}_{16} \mathrm{~F}_{34} \mathrm{NO}_{4} \mathrm{P}$ : C $27.20 ; \mathrm{H} 1.52 ; \mathrm{N}$ 1.32; P 2.92; found: C 27.22; H 1.50; N 1.36; P 2.90 .

Bis [1-(F-nonyl)methyl] monomorpholinophosphate, D(F9C1)MMP. The same procedure as for $\mathrm{D}(\mathrm{F} 8 \mathrm{C} 2) \mathrm{MMP}$, when applied to $1.93 \mathrm{~g}(45.0 \mathrm{mmol})$ of $\mathrm{OPCl}_{3}, 41.84 \mathrm{~g}(90.2 \mathrm{mmol})$ of 1-(F-nonyl)methanol, $11.3 \mathrm{~mL}(8.51 \mathrm{~g}, 90.3 \mathrm{mmol})$ of $\mathrm{NEt}_{3}$ and, subsequently, $7.93 \mathrm{~g}(91.1 \mathrm{mmol})$ of morpholine, yielded $5.42 \mathrm{~g}(37 \%)$ of D(F9C1)MMP. MW $=1131 \mathrm{~g}, \mathrm{m.p} .=82^{\circ} \mathrm{C}$. ${ }^{1} \mathrm{H}$ NMR $\left(\delta, \mathrm{CDCl}_{3}\right): 3.2\left(\mathrm{~m}, 4 \mathrm{H}, \mathrm{CH}_{2} \mathrm{~N}\right), 3.65(\mathrm{~m}, 4 \mathrm{H}$, $\left.\mathrm{CH}_{2} \mathrm{OCH}_{2}\right), 4.2\left[\mathrm{~m}, 4 \mathrm{H},\left(\mathrm{CH}_{2} \mathrm{O}\right)_{2} \mathrm{PO}\right] .{ }^{19} \mathrm{~F}$ NMR $\left(\delta, \mathrm{CDCl}_{3}\right)$ : 
$-81.5\left(\mathrm{CF}_{3}\right),-114.1\left(\mathrm{CF}_{2} \mathrm{CH}_{2}\right),-122.5(4 \mathrm{~F}),-123.4(6 \mathrm{~F})$, $-124.2(2 \mathrm{~F}),-126.5\left(\mathrm{CF}_{3} \mathrm{CF}_{2}\right) ;{ }^{31} \mathrm{P}$ NMR $\left(\delta, \mathrm{CDCl}_{3}\right): 8.35$. IR $\left(\mathrm{v} / \mathrm{cm}^{-1}\right)$ : 1250-1150 $(\mathrm{P}=\mathrm{O}, \mathrm{CF}), 970(\mathrm{PN})$. Anal. calcd (\%) for $\mathrm{C}_{24} \mathrm{H}_{16} \mathrm{~F}_{34} \mathrm{NO}_{4} \mathrm{P}$ : C 25.48; $\mathrm{H}$ 1.07; N 1.24; P 2.90; found: C 25.12; H 0.99; N 1.25 ; P 2.60 .

\section{Results and Discussion}

\section{Synthesis}

The series of (perfluoroalkyl)alkyldimorpholinophosphate surfactants has been extended; the yields were in the $52-73 \%$ range. The procedure consists, in its first step, in the phosphorylation of the fluorinated alcohol by phosphorus oxychloride as shown in Scheme 1. The formation of di- and triesters and the chlorination of the alcohol can be avoided by using dry ether and an excess of triethylamine, which result in the precipitation of triethylammonium chloride. ${ }^{17}$ The (perfluoroalkyl)alkyldimorpholinophosphates were subsequently obtained by reaction with morpholine. $\mathrm{Ca} .5 \%$ of the crude material obtained consisted of a by-product, which was identified by NMR to be the diester $\mathrm{D}(\mathrm{F} n \mathrm{Cm}) \mathrm{MMP}$. No pyrophosphate was detected. The stability of the F $n \mathrm{CmDMPs}$ was assessed by heating aqueous solutions of these compounds (10

$$
2 \mathrm{ROH}+\underset{\mathrm{POH}}{\mathrm{R}=\mathrm{C}_{n} \mathrm{~F}_{2 n+1} \mathrm{C}_{m} \mathrm{H}_{2 m}} \stackrel{\mathrm{NEt}_{3}, \mathrm{Et}_{2} \mathrm{O}}{=\mathrm{C}_{m} \mathrm{H}_{2 m+1}}
$$
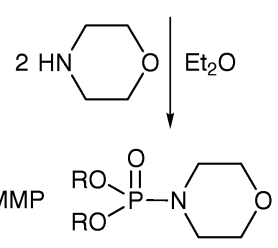

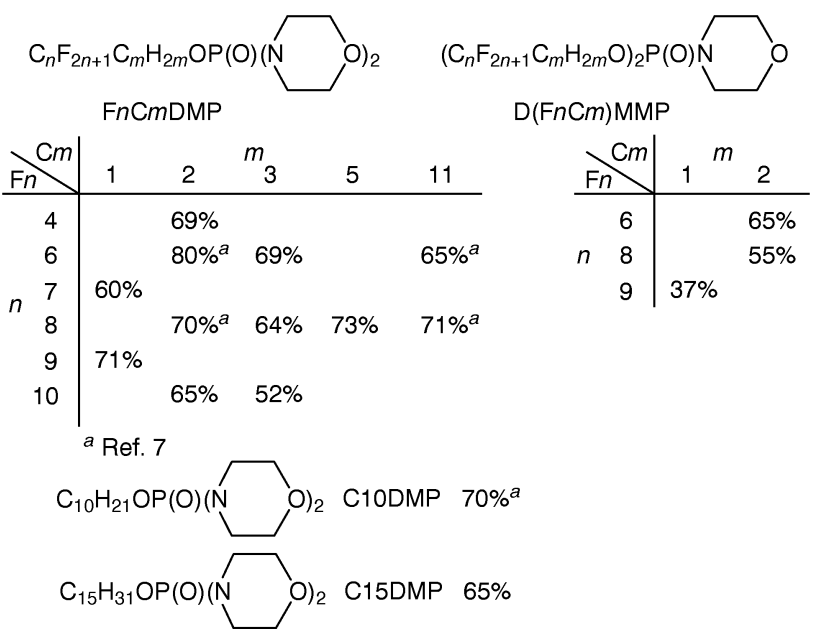

Scheme 1 Synthesis of ( $F$-alkyl)alkyldimorpholinophosphates and bis $[(F$-alkyl)alkyl $]$ monomorpholinophosphates $\mathrm{g} \mathrm{L}^{-1}$ ) for $18 \mathrm{~h}$. After evaporation of the water, the residue was dissolved in $\mathrm{CDCl}_{3}$ and analysed by ${ }^{1} \mathrm{H}$ and ${ }^{31} \mathrm{P}$ NMR. No degradation of the products was detected.

The synthesis of bis $[(F$-alkyl)alkyl $]$ monomorpholinophosphates also involve the direct phosphorylation of the $F$-alkylated alcohols by $\mathrm{OPCl}_{3}$, followed by reaction with morpholine. However, the duration of the first step was increased because of steric crowding due to the presence of two bulky and rigid perfluoroalkylated chains. In the case of $\mathrm{D}(\mathrm{F} 8 \mathrm{C} 2) \mathrm{MMP}$ and $\mathrm{D}$ (F9C1)MMP, heating at reflux was even necessary to achieve phosphorylation of the alcohols. Furthermore, two equivalents of morpholine without triethylamine were needed: it was impossible to obtain the $\mathrm{D}(\mathrm{F} n \mathrm{Cm}) \mathrm{MMPs}$ with only one equivalent of morpholine in the presence of an excess of triethylamine as in the case of the dimorpholinophosphates. This can be explained by a competition between the two bases for the remaining, crowded and less reactive $\mathrm{P}-\mathrm{Cl}$ bond. With the procedure reported here, the $\mathrm{D}(\mathrm{F} n \mathrm{Cm}) \mathrm{MMPs}$ could be obtained in $37-65 \%$ yield.

\section{Surface and interfacial tension measurements}

It is well-known that fluorinated surfactants are much more surface active at the air/water interface than standard hydrogenated surfactants. ${ }^{1-4,18,19}$ We have assessed the surface activity of the perfluoroalkylated mono- and dimorpholinophosphates by measuring the surface tension, $\gamma_{\mathrm{s}}$, of their aqueous solutions and the interfacial tension between these solutions and perfluorodecalin, $\gamma_{i}($ FDC $)$. Long equilibration times (several hours) were necessary, especially for the surfactants with the highest $\mathrm{F} n: \mathrm{Cm}$ ratio, in order to reach equilibrium. ${ }^{20}$ The results are collected in Table 1 . It can be seen that the F $n$ CmDMPs can reduce the surface tension of water from 72 to $21.2-24.5 \mathrm{mN} \mathrm{m}^{-1}$, even when they are very short, as in the case of F4C2DMP. In comparison, the hydrogenated analogues, C10DMP and C15DMP, are much less effective and reduce the $\gamma_{\mathrm{s}}$ of water to $30-32.5 \mathrm{mN} \mathrm{m}^{-1}$ only, even when higher concentrations of the surfactant (e.g. $10^{-4} \mathrm{~mol}$ $\mathrm{L}^{-1}$ ) are used. The length of the hydrophobic chain has only a small impact on the surface tension value at the critical micellar concentration $(\mathrm{cmc})$, in line with other results on nonionic surfactants. ${ }^{21}$ The cmc of the fluorinated products are very low and decrease, as expected, when the degree of fluorination and/or the hydrocarbon spacer length increase. It is wellknown that fluorinated surfactants have a higher tendency to form micelles than hydrogenated analogues. ${ }^{18,19}$ The $\mathrm{cmc}$ value of F8C2DMP is $c a$. two orders of magnitude lower than that of C10DMP. On the other hand, it was found that for a given length of the fluorinated chain, increasing the length of the hydrogenated spacer did not lower the $\mathrm{cmc}$ value as much as would have been expected; see for example the $\mathrm{cmc}$ of F6C2DMP and F6C11DMP $\left(97.3 \times 10^{-6}\right.$ and $25.4 \times 10^{-6}$ $\mathrm{mol} \mathrm{L}^{-1}$, respectively). This observation may indicate that it is the length of the fluorinated chain that actually controls micellization and that the spacer only plays a minor role. ${ }^{22}$

The fluorinated $\mathrm{F} n \mathrm{CmDMPs}$ were also found to reduce the interfacial tension between water and perfluorodecalin very effectively, i.e. from 56 to $c a .1 \mathrm{mN} \mathrm{m}^{-1}$, while their hydrogenated analogues reduce $\gamma_{\mathrm{i}}$ only to $10 \mathrm{mN} \mathrm{m}^{-1}$.

The $\mathrm{D}(\mathrm{F} n \mathrm{Cm}) \mathrm{MMPs}$ were found to be less-surface active than the $F n C m D M P s . D(F 6 C 2) M M P$ reduces the surface tension of water to $23.5 \mathrm{mN} \mathrm{m}^{-1}$ but only at concentrations higher than $1.5 \times 10^{-5} \mathrm{~mol} \mathrm{~L}^{-1}$. Likewise, high concentrations were needed to reduce the interfacial tension between water and perfluorodecalin (FDC) significantly $\left(\gamma_{\mathrm{i}}=12.5 \mathrm{mN}\right.$ $\mathrm{m}^{-1}$ at $\left.10^{-2} \mathrm{~mol} \mathrm{~L}^{-1}\right)$. D(F6C2)MMP being soluble in FDC, a significant amount of the surfactant remains in the form of monomers, and is therefore not available to form the monolayer. The high interfacial tension values obtained with $\mathrm{D}$ (F8C2)MMP and D(F9C1)MMP $\left(\gamma_{\mathrm{i}}=15.5 \mathrm{mN} \mathrm{m}^{-1}\right.$ and 
Table 1 Surface and interfacial tensions of aqueous solutions of perfluoroalkylated dimorpholinophosphates and monomorpholinophosphates at $25^{\circ} \mathrm{C}$ and biological data

Acute toxicity in mice $\mathrm{LD}_{50}$

\begin{tabular}{|c|c|c|c|c|c|c|c|c|}
\hline Compound & $\begin{array}{l}\mathrm{cmc} / \\
\mathrm{mol} \mathrm{L}\end{array}$ & $\begin{array}{l}\gamma_{\mathrm{s}} / \\
\mathrm{mN} \mathrm{m}^{-1}\end{array}$ & $\begin{array}{l}\mathrm{c} / \\
\mathrm{mol} \mathrm{L}\end{array}$ & $\begin{array}{l}\gamma_{\mathrm{i}}(\mathrm{FDC}) / \\
\mathrm{mN} \mathrm{m}^{-1}\end{array}$ & $\begin{array}{l}\text { i.v./ } \\
\text { mg (kg body weight })^{-1}\end{array}$ & Survival & $\begin{array}{l}\text { i.p./ } \\
\text { g (kg body weight })^{-1}\end{array}$ & Survival \\
\hline F4C2DMP & $3.2 \times 10^{-3}$ & 24.5 & $8.3 \times 10^{-3}$ & 3.7 & $>500$ & $10 / 10$ & - & - \\
\hline F6C2DMP & $1.0 \times 10^{-4}$ & 22.0 & $3.2 \times 10^{-3}$ & 2.3 & $>250$ & $10 / 10$ & - & - \\
\hline F6C11DMP & $2.6 \times 10^{-5}$ & 23.5 & $2.8 \times 10^{-4}$ & 0.9 & $>25$ & $10 / 10$ & - & - \\
\hline F7C1DMP & $1.5 \times 10^{-5}$ & 21.2 & $2.0 \times 10^{-3}$ & 3.7 & - & - & - & - \\
\hline F8C2DMP & $2.0 \times 10^{-6}$ & 22.5 & $1.5 \times 10^{-4}$ & 0.9 & $>25$ & $10 / 10$ & $>2$ & $10 / 10$ \\
\hline F8C5DMP & $8.9 \times 10^{-7}$ & 22.5 & $6.9 \times 10^{-5}$ & 3.8 & - & - & $\approx 2$ & $5 / 10$ \\
\hline $\mathrm{F}^{2 C 11 \mathrm{DMP}}{ }^{a}$ & $1.0 \times 10^{-6}$ & 23.0 & - & - & $>2000$ & $10 / 10$ & $\approx 4$ & $5 / 10$ \\
\hline F9C1DMP & $7.7 \times 10^{-7}$ & 23.0 & $2.5 \times 10^{-3}$ & 0.9 & - & - & - & - \\
\hline $\mathrm{F} 10 \mathrm{C} 2 \mathrm{DMP} \mathrm{P}^{b}$ & $1.0 \times 10^{-6}$ & 23.5 & - & - & - & - & - & - \\
\hline C10DMP & $2.5 \times 10^{-4}$ & 30.0 & - & - & $>140$ & $10 / 10$ & - & - \\
\hline C15DMP & $1.6 \times 10^{-6}$ & 32.5 & $5.4 \times 10^{-5}$ & 9.4 & - & - & - & - \\
\hline $\mathrm{D}(\mathrm{F} 6 \mathrm{C} 2) \mathrm{MMP}$ & $1.5 \times 10^{-5}$ & 23.5 & $1.7 \times 10^{-2}$ & 12.7 & - & - & $>4$ & $8 / 10$ \\
\hline $\mathrm{D}(\mathrm{F} 8 \mathrm{C} 2) \mathrm{MMP}^{b}$ & $2.1 \times 10^{-6}$ & 23.5 & $1.0 \times 10^{-2}$ & 15.5 & - & - & - & - \\
\hline $\mathrm{D}$ (F9C1)MMP ${ }^{b}$ & $1.0 \times 10^{-5}$ & 25.3 & $9.9 \times 10^{-3}$ & 14.5 & - & - & $>2$ & $9 / 10$ \\
\hline
\end{tabular}

$\gamma_{\mathrm{i}}=14.5 \mathrm{mN} \mathrm{m}^{-1}$ at $10^{-2} \mathrm{~mol} \mathrm{~L} \mathrm{~L}^{-1}$ ), which are very soluble in the fluorocarbon phase, confirm this view. The quasiinsolubility of the monomorpholinophosphates in water explains that $\mathrm{D}(\mathrm{F} 8 \mathrm{C} 2) \mathrm{MMP}$ and $\mathrm{D}(\mathrm{F} 9 \mathrm{C} 1) \mathrm{MMP}$ reduce the surface tension of water to 23.5 and $25.3 \mathrm{mN} \mathrm{m}^{-1}$, respectively, but only at $60^{\circ} \mathrm{C}$.

\section{Acute toxicity and hemolysis data}

The preliminary biological tests performed on the new perfluoroalkylated amphiphiles are collected in Table 1. Dispersions of F6C2DMP, F6C11DMP, F8C2DMP and F8C11DMP (0.05 to $0.5 \mathrm{~g} \mathrm{~L}^{-1}$ in $0.9 \%$ of $\mathrm{NaCl}$ ) did not perturb the growth and viability of Namalva strain lymphoblastoid cell cultures, while a solution of the non-fluorinated analogue C10DMP $\left(0.05 \mathrm{~g} \mathrm{~L}^{-1}\right)$ totally inhibited cell growth and viability. ${ }^{7}$ Likewise, solutions of the fluorinated surfactants did not show any hemolytic activity on a suspension of human red blood cells: in contrast, a solution of C10DMP $\left(0.5 \mathrm{~g} \mathrm{~L}^{-1}\right)$ was strongly hemolytic.

Aqueous dispersions of the above fluorinated surfactants were well-tolerated when administered intravenously (tail vein) in mice; the i.v. $\mathrm{LD}_{50}$ values were higher than $200-250$ $\mathrm{mg}$ ( $\mathrm{kg}$ body weight $)^{-1}$ (10/10 survivals) for F6C2DMP and F8C2DMP (limit of protocol/solubility). To increase the amount of surfactant that could be administered, we prepared a dispersion of the poorly water-soluble F8C11DMP in $0.9 \%$ $\mathrm{NaCl}$ using egg yolk phospholipids. The toxicity tests indicated a $\mathrm{LD}_{50}$ value higher than $2 \mathrm{~g}$ ( $\mathrm{kg}$ body weight) ${ }^{-1}$ (limit of protocol, $10 / 10$ survivals).

Intraperitoneal administration in mice of $\mathrm{F} n \mathrm{CmDMPs}$ in perfluorooctyl bromide (PFOB) gave $\mathrm{LD}_{50}$ values higher than $2 \mathrm{~g}(\mathrm{~kg} \text { body weight })^{-1}$ for F8C2DMP (10/10 survivals) and around $4 \mathrm{~g}$ ( $\mathrm{kg}$ body weight $)^{-1}$ for F8C11DMP $(5 / 10$ survivals). Very encouraging results have also been obtained for the bis-perfluoroalkylated monomorpholinophosphates with i.p. $\mathrm{LD}_{50}$ values larger than 4 and $2 \mathrm{~g}(\mathrm{~kg}$ body

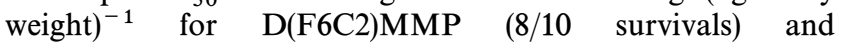
D(F9C1)MMP (9/10 survivals), respectively.

All these results confirm that in spite of their high surface activity, fluorinated morpholinophosphates are well tolerated by cell cultures, are non-hemolytic, and present low to very low acute toxicity, even when administered intravascularly. They confirm our opinion that perfluoroalkylated morpholinophosphates have potential as components of colloidal systems intended for biomedical applications.

\section{Acknowledgements}

Atochem (Pierre-Bénite, France), Alliance Pharmaceuticals Corp. (San Diego, CA, USA) and the Hungarian Scientific Research Foundation (OTKA, No T 022169).

\section{References}

1 J. Greiner, J. G. Riess and P. Vierling, in Organofluorine Compounds in Medicinal Chemistry and Biomedical Applications, eds. R. Filler, T. Kobayashi and L. M. Yagupolski, Elsevier, Amsterdam, 1993, pp. 339-380.

2 J. G. Riess, New J. Chem., 1995, 19, 891.

3 E. Kissa, Fluorinated Surfactants, Surfactant Science Series, M. Dekker, New York, 1993. vol. 50.

4 J. C. Ravey and M. J. Stébé, Colloids Surf., 1994, 84, 11.

5 J. G. Riess, Colloids Surf., 1994, 84, 33.

6 J. G. Riess, F. Frézard, J. Greiner, M. P. Krafft, C. Santaella, P. Vierling and L. Zarif, in Handbook on Non-medical Applications of Liposomes, ed. Y. Barenholz and D. D. Lasic, CRC Press, Boca Raton, FL, 1996, vol. III, ch. 8.

7 M. P. Krafft, P. Vierling and J. G. Riess, Eur. J. Med. Chem., 1991, 26, 545.

8 V. M. Sadtler, M. P. Krafft and J. G. Riess, Angew. Chem., Int. Ed. Engl., 1996, 35, 1976.

9 M. P. Krafft, F. Giulieri, V. M. Sadtler and J. G. Riess, Phosphorus Sulfur Silicon, 1996, 109/110, 281.

10 F. Giulieri, M. P. Krafft and J. G. Riess, Angew. Chem., Int. Ed. Engl., 1994, 33, 1514.

11 Y. Ferro, F. Giulieri and M. P. Krafft, in preparation.

12 N. O. Brace, J. Org. Chem., 1962, 27, 4491.

13 M. Takahashi, Y. Nagasaki and S. Fujii, Jpn. Pat. 02,169,528, 1990; CA, 1993, 113, P 190741q.

14 M. Kotora, M. Hajek, B. Ameduri and B. Boutevin, J. Fluorine Chem., 1994, 68, 49; J. O. Metzger, R. Mahler and A. Schmidt, Liebigs Ann. Org. Bioorg. Chem., 1996, 5, 693; Z. Szlávik and J. Rábai, in preparation.

15 M. Le Blanc, J. G. Riess, D. Poggi and R. Follana, Pharm. Res., 1985, 5, 246.

16 J. G. Riess, S. Pace and L. Zarif, Adv. Mater., 1991, 3, 249.

17 W. J. Hansen, R. Murari and Y. Wedmid, Lipids, 1982, 17, 453.

18 H. Kunieda and K. Shinoda, J. Phys. Chem., 1976, 80, 2468.

19 P. Mukerjee and T. Handa, J. Phys. Chem., 1981, 85, 2292.

20 N. Laakel, P. Rubini, L. Rodehüser and J. J. Delpuech, New J. Chem., 1993, 16, 809.

21 J. C. Ravey, A. Gherbi and M. J. Stébé, Prog. Colloid Polym. Sci., 1988, 76, 234.

22 V. M. Sadtler, F. Giulieri, M. P. Krafft and J. G. Riess, Chem. Eur. $J .$, in the press.

Received in Orsay, France, 20th May 1997; revised $\mathrm{m} / \mathrm{s}$ received 18th November 1997; Paper 7/08757H 